\title{
Idioventricular rhythm complicating acute myocardial infarction
}

\author{
R. M. Norris ${ }^{1}$, C. J. Mercer, and S. E. Yeates \\ From Green Lane Hospital, Auckland, New Zealand
}

The incidence, natural history, prognosis, and electrocardiographic characteristics of idioventricular rhythm complicating acute myocardial infarction are described. It occurred as a transient arrhythmia nearly always within 24 hours of infarction in 61 ( $8 \%$ ) of 737 patients, and was characterized by paroxysms of between 6 and 20 beats with widened bizarre $Q R S$ complexes at a rate of between 60 and 90 a minute. Most cases showed fusion beats and $P$ waves dissociated from the $Q R S$ complexes, and in many cases idioventricular rhythm started during the slow phase of sinus arrhythmia. Though it usually occurred in patients with moderately severe transmural infarcts, the incidence of ventricular fibrillation and subsequent mortality was no greater than in patients with infarcts of equivalent severity who did not have idioventricular rhythm. It is concluded that this rhythm is a common and relatively benign arrhythmia complicating myocardial infarction, and that it should be distinguished from ventricular tachycardia.

A major development after the introduction of coronary care units has been the appreciation of the prognostic significance of ventricular arrhythmias occurring early after acute myocardial infarction. Frequent ventricular premature beats, salvoes of extrasystoles, ventricular tachycardia, and ventricular ectopic beats showing the $R$ on $T$ phenomenon, have all been shown to be precursors of ventricular fibrillation, and to warrant aggressive treatment with anti-arrhythmic drugs (Lown et al., 1967). Recently, however, a benign transient ventricular arrhythmia, with a rate slower than ventricular tachycardia, has been described, and named idioventricular rhythm (Rothfeld et al., 1968). The purpose of the present report is to describe further the natural history of idioventricular rhythm as seen in a coronary care unit, and to record its incidence, prognostic significance, and electrocardiographic characteristics.

\section{Patients and methods}

Between March 1967 and December 1969, 737 patients with acute myocardial infarction were admitted to a coronary care unit (Norris, 1968). Myocardial infarction was presumed to have occurred when at least two of the following three criteria were satisfied. (I) Characteristic clinical presentation. (2) Pathological $Q$ waves, ST elevation, or $T$ wave inversion in the electrocardio-

Received 26 February 1970.

1 Present address: M.R.C. Cardiovascular Research Unit, Royal Postgraduate Medical School, London. gram, with evolutionary changes. (3) A rise in serum aspartate aminotransferase to over 40 units $/ \mathrm{ml}$.

Patients stayed in the unit for a mean period of three days, and 53 per cent were admitted within six hours of onset of the infarct. While in the unit, ro-second electrocardiogram strips were taken by nurses hourly, or whenever an arrhythmia was seen on a central oscilloscope screen. These records were subsequently studied, the data transferred to I.B.M. punch cards, and an analysis made of the types of arrhythmia which occurred.

\section{Results}

An analysis of ventricular arrhythmias showed that in addition to ventricular ectopics occurring singly or in salvoes, and ventricular tachycardia at a rate of over I00 a minute, a transient rhythm characterized by runs of bizarre widened QRS complexes, which were slightly irregular and came in runs of between 6 and 20 beats, occurred in 61 patients. The rate was between 60 and 90 a minute, and was always about the same as the sinus rate at the time the arrhythmia occurred (Fig. I). This idioventricular rhythm was clearly differentiated from ventricular tachycardia by its slower rate. It nearly always occurred within 24 hours of the onset of infarction, and frequently during sleep. Cases of acute pump failure (Lown et al., 1967) in which widened bizarre QRS complexes occurred as a terminal event are excluded from this study. 


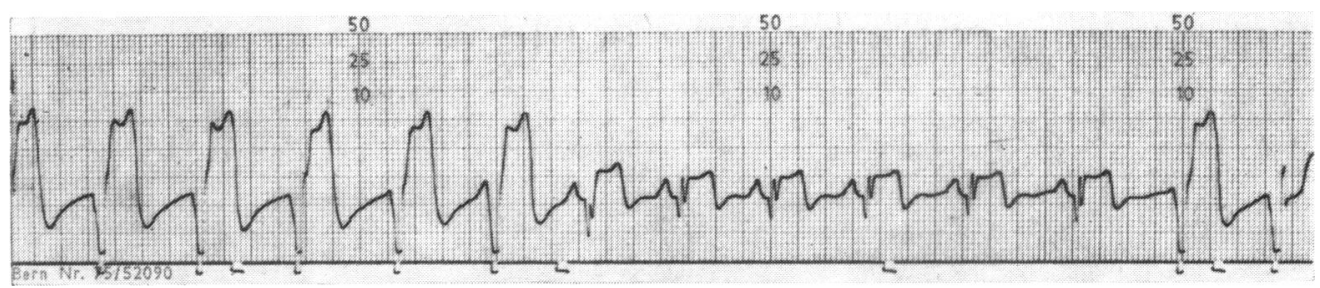

FIG. I The finish of one and the start of another episode of idioventricular rhythm have been recorded, with six beats of sinus rhythm intervening. The QRS complexes of idioventricular rhythm are widened and bizarre, the rate ( 82 a minute) is similar to the sinus rate, and the start of the second episode occurs during the slow phase of sinus arrhythmia. A P wave is visible before the fifth and sixth beats of the first episode, and the seventh beat is a fusion beat.

A comparison of the incidence in the various types of infarction of ventricular tachycardia and idioventricular rhythm is given in Tables I and 2, which show that the two arrhythmias had an approximately equal incidence, and that both occurred much more frequently in transmural (infarction with pathological $Q$ waves) than in subendocardial infarction (infarction with ST and $T$ wave changes only). There was a tendency for ventricular tachycardia to be commoner in anterior and idioventricular tachycardia in posterior infarction, but this was not statistically significant. Whereas ventricular tachycardia carried a high mortality and was associated with ventricular

TABLE I Extent of infarction in patients with idioventricular rhythm (IVR) and ventricular tachycardia $(V T)$

\begin{tabular}{lccc}
\hline Type of infarct & $\begin{array}{l}\text { No. of } \\
\text { cases }\end{array}$ & $\begin{array}{l}\text { No. with } \\
\text { IVR }\end{array}$ & $\begin{array}{l}\text { No. with } \\
\text { VT }\end{array}$ \\
\hline Transmural & 459 & $53(12 \%)$ & $59(13 \%)$ \\
Subendocardial & 183 & $3(2 \%)$ & $9(5 \%)$ \\
Indefinite & 95 & $5(5 \%)$ & $17(18 \%)$ \\
\hline Total & 737 & $61(8 \%)$ & $85(11 \%)$
\end{tabular}

* Includes cases of combined anterior and posterior infarction and left bundle-branch block.

TABLE 2 Site of infarction in patients with idioventricular rhythm (IVR) and ventricular tachycardia $(V T)$

\begin{tabular}{lccc}
\hline Site of infarct & $\begin{array}{l}\text { No. of } \\
\text { cases }\end{array}$ & $\begin{array}{l}\text { No. with } \\
\text { IVR }\end{array}$ & $\begin{array}{l}\text { No. with } \\
\text { VT }\end{array}$ \\
\hline Anterior & 384 & $25(7 \%)$ & $45(12 \%)$ \\
Posterior & 258 & $31(12 \%)$ & $23(9 \%)$ \\
Indefinite & 95 & $5(5 \%)$ & $17(18 \%)$ \\
\hline Total & 737 & $61(8 \%)$ & $85(11 \%)$
\end{tabular}

* Includes cases of combined anterior and posterior infarction and left bundle-branch block. fibrillation in over half the cases, the mortality and occurrence of ventricular fibrillation in association with idioventricular rhythm was no greater than would be expected in this group of patients with moderately severe transmural infarcts (Table 3). Idioventricular rhythm nearly always occurred during the first 24 hours after onset of infarction, while ventricular tachycardia, though commonest at the onset, also occurred after the first day.

Electrocardiographic characteristics of idioventricular rhythm are shown in Fig. I to 3. In most cases, it started during the slow phase of sinus arrhythmia, with a rate slightly faster than the sinus rate at the time, and persisted for 6 to 20 beats. The beats were widened and bizarre in appearance, and fusion beats often occurred as the first or last beat of the paroxysm. P waves which were dissociated from the QRS complexes, and of similar contour to sinus $P$ waves, were frequently seen during idioventricular rhythm (Fig. 2). When sinus rhythm was restored, the rate was usually a little faster than immediately before idioventricular rhythm occurred, and frequently a further episode of slowing would be followed by another paroxysm of idioventricular rhythm (Fig. 3). In one case, idioventricular rhythm was followed a few hours later by an episode of ventricular parasystole, the parasystolic beats again being of the same contour as the idioventricular rhythm beats.

In order to explore further the association

TABLE 3 Complications occurring in patients with idioventricular rhythm and ventricular tachycardia

\begin{tabular}{lcl}
\hline & $\begin{array}{l}\text { Idioventricular } \\
\text { rhythm }\end{array}$ & $\begin{array}{l}\text { Ventricular } \\
\text { tachycardia }\end{array}$ \\
\hline Total cases & $6 \mathbf{I}$ & 85 \\
Ventricular fibrillation & $8(13 \%)$ & $45(53 \%)$ \\
Mortality & $10(16 \%)$ & $44(52 \%)$ \\
\hline
\end{tabular}




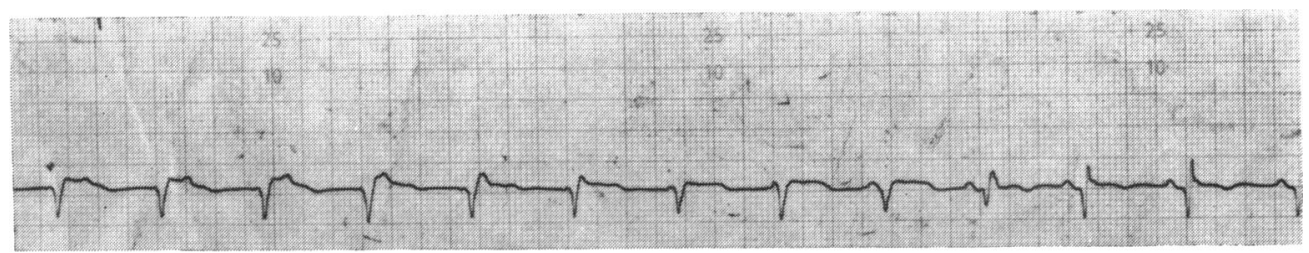

FIG. 2 A short episode of idioventricular rhythm occurring in a 56-year-old man 12 hours after an anterior infarct. This record shows clear evidence of atrioventricular dissociation, the atrial rate being 86-92 a minute, and the ventricular rate 84 a minute. The last three beats are sinus beats, and the fourth to last is a fusion beat. In this case the ventricles were recaptured by an increase in rate of the sinus node.

FIG. 3 Idioventricular rate occurring in a 37-year-old man who had sustained an anterior myocardial infarction four hours previously. This record is continuous. Three paroxysms of idioventricular rhythm are shown, together with the start of a fourth. The paroxysms of idioventricular rhythm are separated by a few beats of sinus rhythm showing obvious sinus arrhythmia with the slow phase immediately preceding idioventricular rhythm. The sixth beat in the first row, the seventh beat in the second row, and the second beat in the third row are fusion beats. $P$ waves having a contour similar to that of sinus $P$ waves, and a rate almost identical to that of the idioventricular rhythm, are seen during the first and second paroxysms.
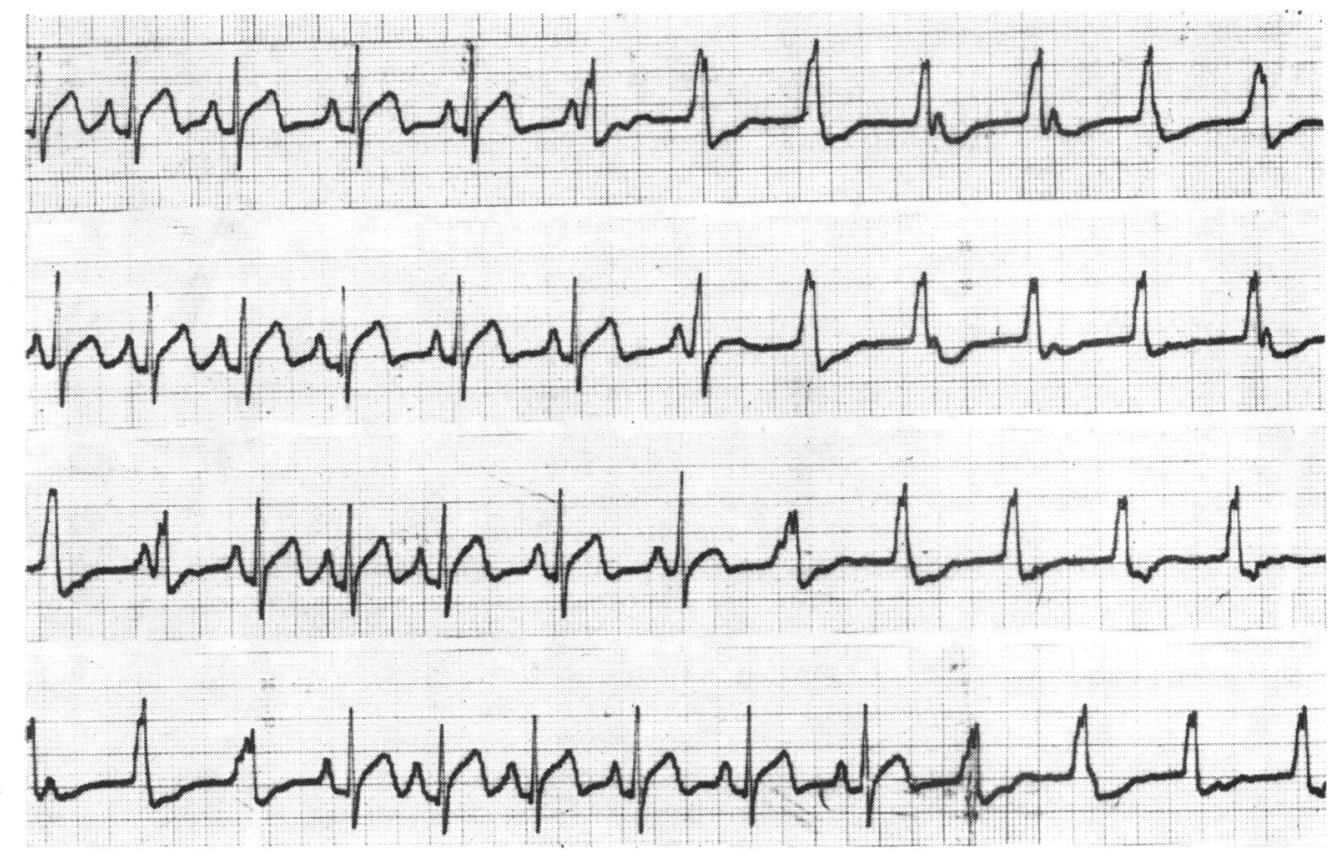

between sinus arrhythmia and idioventricular rhythm, and to document the evidence for the ventricular origin of the rhythm, the records of all patients were examined for atrioventricular dissociation, fusion beats, ventricular ectopic beats of the same configuration as the idioventricular rhythm beats, and sinus arrhythmia immediately preceding or following the episodes of idioventricular rhythm. Sinus arrhythmia was considered to be present when there was a change in rate of Io per cent or more between the two first and two last beats of between 3 and 12 sinus beats immediately preceding or following idioventricular rhythm. Where sinus arrhythmia was present, it was noted whether the rate was getting slower immediately before the onset of idioventricular rhythm, and whether it started rapidly and then slowed off with the resumption of sinus rhythm after the paroxysm of idioventricular rhythm. These results are shown in Table 4. Fusion beats and evidence of atrioventricular dissociation were present in two-thirds of cases, and ventricular 
TABLE 4 Electrocardiographic characteristics of idioventricular rhythm

Total number of cases

Fusion beats during idioventricular rhythm

Dissociated $\mathbf{P}$ waves during idioventricular rhythm

Sinus arrhythmia immediately preceding or following idioventricular rhythm

Ventricular ectopics of same configuration as idioventricular rhythm beats

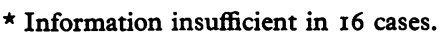

ectopic beats of the same configuration as the idioventricular rhythm beats were present in a half. According to the definition used, sinus arrhythmia was present immediately before or after idioventricular rhythm in over half of the cases in which information was available, and in these cases idioventricular rhythm nearly always started during the slow phase with restoration of sinus rhythm in the rapid phase. In a randomly selected group of patients who had infarcts of equivalent severity not complicated by idioventricular rhythm, sinus arrhythmia occurred at some time during the first 24 hours after admission in onethird, but was by no means continuous over the whole 24 -hour period.

\section{Discussion}

Since the introduction of continuous electrocardiogram monitoring for acute myocardial infarction, much has been written about the incidence and prognostic significance of ventricular arrhythmias, particularly ventricular tachycardia (Spann et al., I946; Bashour, Jones, and Edmonson, 1967; Fluck et al., 1967; Lown et al., 1967; Kitchell, 1966; Raftery et al., 1969). Most authors have agreed that ventricular tachycardia occurs in about Io per cent of patients after infarction and carries a poor prognosis, principally because of its frequent association with ventricular fibrillation. Some workers have also commented on the occasional occurrence of an arrhythmia similar to ventricular tachycardia, but having a rate of less than 100 a minute. No firm opinion on the prognostic significance of this arrhythmia was given, though Bashour et al. (1967) and Raftery et al. (1969) have regarded it as a potential forerunner of ventricular fibrillation which should be suppressed by anti-arrhythmic drugs.

The first description of idioventricular rhythm as a common arrhythmia occurring after infarction, and a separate entity from ventricular tachycardia, was made by Rothfeld and his associates in 1968 . These workers described idioventricular rhythm as an ectopic ventricular rhythm with a rate between 60 and 100 a minute which was transient, and only lasted between 4 and 30 beats. Using a relay telemetric system for constant monitoring, they found that the arrhythmia occurred in 36 of 100 consecutive patients with acute myocardial infarction. It usually occurred during sinus bradycardia or the slow phase of sinus arrhythmia, and unlike ventricular tachycardia it did not progress to ventricular fibrillation, nor did it influence the prognosis adversely.

The present study agrees in most respects with that of Rothfeld et al., and it is probable that the considerably lower incidence of idioventricular rhythm found by us is due to the fact that we were not using a constant recording device, so that some cases of this very transient arrhythmia may not have been recorded. This is probably the reason that idioventricular rhythm has been described so infrequently in the past, even from units practising continuous electrocardiographic monitoring and intermittent recording.

The present study agrees with that of Rothfeld and his colleagues, in the finding that idioventricular rhythm frequently started during the slow phase of sinus arrhythmia, but disagrees in that it seldom occurred during sinus bradycardia. Though not all cases started during sinus arrhythmia, and considerable sinus arrhythmia frequently occurred without idioventricular rhythm, it did appear that a change in sinus rate from faster to slower was one factor which precipitated it in a patient who was predisposed to it. Since idioventricular rhythm nearly always occurred during the first 24 hours after a moderately severe transmural infarct, it seems likely that infarcted ventricular muscle was the major factor causing it. The predisposing factor of a slowing in sinus rate, however, and frequent interaction of sinus and idioventricular pacemakers, suggest that the mechanism might be similar to that of ventricular parasystole. It was of interest that in one case, ventricular parasystole from a similar ventricular focus occurred about 12 hours after idioventricular rhythm. It is planned to study the relation between sinus arrhythmia and idioventricular rhythm further by using carotid sinus pressure and intravenous atropine to change the sinus rate. Preliminary studies on one patient at the time of occurrence of idioventricular rhythm showed a tendency for the arrhythmia to recur with carotid sinus pressure.

It is well known that differentiation of ventricular from supraventricular arrhythmias with aberrant intraventricular conduction is 
often difficult and sometimes impossible (Kistin, 1966). In particular the presence of atrioventricular dissociation is not necessarily an indication that an arrhythmia is ventricular, as an arrhythmia originating in the atrioventricular node can show both aberrant conduction of the QRS complexes and atrioventricular dissociation (Kistin, 1966; Massumi, Tawakkol, and Kistin, 1967). The presence of fusion beats is, however, a reliable indication of a ventricular origin (Pick and Langendorf, 1968), though again exceptions have been described (Kistin, 1966). The occurrence of fusion beats in two-thirds of the cases in this series, together with the frequent occurrence of ventricular ectopic beats having the same configuration as the idioventricular rhythm beats, provides good evidence that it is a ventricular arrhythmia, and not an example of nodal rhythm with aberrant intraventricular conduction. A further possibility is that idioventricular rhythm is a type of ventricular tachycardia showing exit block of varying degree (Gazes, 1966; Lown et al., 1967). This is considered to be unlikely, as obvious changes in rate were not seen in cases of idioventricular rhythm, and the association of this rhythm with ventricular tachycardia in the same patient was no more frequent than could have occurred by chance.

The benign nature of idioventricular rhythm and its lack of association with ventricular fibrillation suggests that prolonged anti-arrhythmic treatment and electrocardiographic monitoring are not necessary for patients in whom it is observed. Since the arrhythmia usually occurs early in the course of moderately severe transmural infarction, it has been our practice to treat these patients with intravenous lignocaine in a dose of $I$ to 3 mg. a minute for one to two days, and to continue oral procainamide in a dose of $500 \mathrm{mg}$. 6-hourly for the duration of the hospital admission. That the relatively uncommon occurrence of ventricular fibrillation in patients with idioventricular rhythm is unlikely to be due to the anti-arrhythmic treatment is, however, shown by the much higher incidence of ventricular fibrillation in patients with ventricular tachycardia, who had as much or more anti-arrhythmic treatment (Table 3). Though the administration of anti-arrhythmic drugs may be a wise precaution in all ventricular arrhythmias occurring after infarction, it is nevertheless of great clinical importance to distinguish idioventricular rhythm from other ventricular arrhythmias so that the patient and his relatives can be reassured, and prolonged electrocardiographic monitoring avoided.

\section{References}

Bashour, F. A., Jones, E., and Edmonson, R. (1967) Cardiac arrhythmias in acute myocardial infarction II. Incidence of the common arrhythmias with special reference to ventricular tachycardia. Diseases of the Chest, 51, 520 .

Fluck, D. C., Olsen, E., Pentecost, B. L., Thomas, M., Fillimore, S. J., Shillingford, J. P., and Mounsey, J. P. D. (1967). Natural history and clinical significance of arrhythmias after cardiac infarction. British Heart fournal, 29, 170.

Gazes, P. (1966). In The Current Status of Intensive Coronary Care, p. 71. Ed. by the American College of Cardiology. The Charles Press, New York.

Kistin, A. D. (1966). Problems in the differentiation of ventricular arrhythmia from supraventricular arrhythmia with abnormal QRS. Progress in Cardiovascular Disease, 9, I.

Kitchell, J. R. (1966). In The Current Status of Intensive Coronary Care, p. 46. Ed. by the American College of Cardiology. The Charles Press, New York.

Lown, B., Fakhro, A. M., Hood, W. B., and Thorn, G. W. (1967). The coronary care unit. Fournal of the American Medical Association, 199, 188.

Massumi, R. A., Tawakkol, A. A., and Kistin, A. D. (1967). Reevaluation of electrocardiographic and bedside criteria for diagnosis of ventricular tachycardia. Circulation, 36, 628.

Norris, R. M. (1968). Acute coronary care. New Zealand Medical fournal, 67, 470.

Pick, A., and Langendorf, R. (1968). Recent advances in the differential diagnosis of $A-V$ junctional arrhythmias. American Heart fournal, 76, 553.

Raftery, E. B., Rehman, M. F., Banks, D. C., and Oram, S. (1969). Incidence and management of ventricular arrhythmias after acute myocardial infarction. British Heart fournal, 31, 273.

Rothfeld, E. L., Zucker, I. R., Parsonnet, V., and Alinsonorin, C. A. (1968). Idioventricular rhythm in acute myocardial infarction. Circulation, 37, 203.

Spann, J. F., Moellering, R. C., Haber, E., and Wheeler, E. O. (1964). Arrhythmias in acute myocardial infarction. New England fournal of Medicine, 271, 427 .

\section{Addendum}

Since this paper was written, Schamroth (I969) has drawn attention to the confused nomenclature of this arrhythmia, and has suggested the term 'idioventricular tachycardia'. Another descriptive term is 'accelerated ventricular rhythm' (Marriott and Menendez, I966). Of these two, the latter seems to us to be preferable, as the arrhythmia is not a tachycardia in the ordinary sense.

\section{References}

Marriott, H. J. L., and Menendez, M. M. (1966) A-V dissociation revisited. Progress in Cardiovascular Disease, 8, 522.

Schamroth, L. (1969). Idioventricular tachycardia. Diseases of the Chest, 56, 466. 